\title{
Categorisation of designs according to preference values for shape rules
}

\author{
Sungwoo Lim ${ }^{1}$, Miquel Prats ${ }^{2}$, Scott Chase ${ }^{1}$ and Steve Garner ${ }^{2}$ \\ ${ }^{1}$ University of Strathclyde, UK, ${ }^{2}$ The Open University, UK
}

\begin{abstract}
Shape grammars have been used to explore design spaces through design generation according to sets of shape rules with a recursive process. Although design space exploration is a persistent issue in computational design research, there have been few studies regarding the provision of more preferable and refined outcomes to designers. This paper presents an approach for the categorisation of design outcomes from shape grammar systems to support individual preferences via two customised viewpoints: (i) absolute preference values of shape rules and (ii) relative preference values of shape rules with shape rule classification levels with illustrative examples.
\end{abstract}

\section{Introduction}

One valuable technique to conceive designs is to generate design alternatives. Computational advancements and the evolution of modern design processes have opened new lines of research based on generative systems. The purpose of generative systems is not always to reach a unique optimal solution but instead to display a range of design alternatives. There are many different variants of generative design systems. They typically generate satisfactory designs starting from little or nothing, being guided by performance criteria within a given design space [1]. One way of obtaining sets of satisfactory designs is to define preference values for generative rules. In other words, instead of randomly generating lots of designs and then looking for meaningful solutions, it is sometimes more reasonable to define rules that generate only sequences of designs that are accord with designer preferences.

Shape grammars [2] are production systems that generate designs according to sets of shape rules. These rules are of the form $a \rightarrow b$, where $a$ 
and $b$ are both labelled shapes, and are applicable to a shape $S$ if there is a transformation that imbeds $a$ in $S$. A shape rule is applied by replacing the transformed shape $a$ in $S$ with the similarly transformed shape $b$. These allow the construction of complex shapes from simple shape elements. The potential for applying shape grammars to explore design spaces has been applied in areas such as architectural and consumer product design [3]. Despite a history going back decades [4], progress in computer implementation of shape grammar systems has been slow [5]. This is partly due to complexities in object representation used in such systems [6] but is also possibly a consequence of the characteristic of producing large, possibly an infinite number of outcomes [7]. As a result relatively few researchers have attempted to categorise outcomes from shape grammar systems. While diversity and number of outcomes may be appreciated by designers, they may wish to limit this number in order to reduce their efforts to find preferable (or appropriate) ones.

The research described here results from an ongoing project concerning design synthesis and shape generation (DSSG). The project explores how designers generate shapes and how shape computation systems might support designers without impinging upon their creativity. The aim of this paper is to present an approach to categorising design outcomes from shape grammar systems to support individual preference. It offers the possibility of providing more preferable and refined outcomes to designers based on their own ways of shape generation. Here, the categorisation of design outcomes is not intended to reflect a measure of similarity or style but instead is intended to reflect the likelihood that designs would be produced by a designer. This likelihood is based on experimental data concerned with analysing how designers specify and manipulate shapes when exploring designs [8]. This analysis led to the definition of shape rules believed to capture the manipulations typically used by designers, and to data related to the frequency that such rules were used to explore designs.

\section{Clustering via customisable viewpoints}

Shape rules can formalise the creative process that involves the generation of designs, the selection of the preferable, and the seeding of a new generation, until a competent design is found or the entire design space has been explored [9]. This process, however, may not be ideal for design exploration since design spaces tend to be immense and the probability of obtaining a satisfactory design in a reasonable length of time is very small. 
One possible way to customise design outcomes is by categorisation according to the similarity of shape characteristics. Clusters of designs may be organised into a hierarchical structure where they are broken down into subclusters [10]. In this case, a hierarchical classifier is needed to divide the classes into contextual subgroups, which are then further divided to produce a tree structure defining relationships between classes [11]. A number of methods are extant for hierarchical clustering depending on the area of application, e.g. in biological taxonomy, psychology and cognitive science [12], physics [13], and artificial intelligence [14].

Some investigations have been conducted into multiple viewpoints for clustering. Researchers have found that different results can be obtained when the same data set is analysed using a different clustering strategy during computational clustering [15, 16]. For example, Howard-Jones [17] carried out an experiment in which subjects looked at a geometrical shape, generating as many interpretations of the shape as possible based on different viewpoints. Duffy and Kerr [18] suggest that designers require different viewpoints from past designs and abstractions in order to facilitate the effective utilisation of past design knowledge, and pointed out the need to support different viewpoints, termed 'Customised Viewpoints' $(\mathrm{CV})$. Manfaat and Duffy [19] extended this theory to support the effective utilisation of spatial layouts for ship design by hierarchical levels of abstractions according to designers' needs. To maximise the capability of $C V$, the selection of criteria for clustering that are appropriate to the data being investigated is crucial [20].

Due to the characteristics of Shape Grammar systems, which potentially produce large numbers of outcomes [7], categorising design outcomes could facilitate more widespread use of this design paradigm. As the main aim of $C V$ is to classify designs via different viewpoints, adapting the concept of $C V$ could provide a way of categorising and refining outcomes by individual viewpoints and preferences.

\section{Preference values and classification of shape rules}

Understanding designers' preferences when interacting with shapes is needed to utilise $C V$ with shape grammars. As a part of the DSSG project, a sketch observation experiment [8] to identify shape rules in shape transformation was undertaken. Six architects and eight product designers with various ranges of professional experience were involved in the experiment. They responded to a series of conceptual design tasks and produced an output of nearly 300 sketches. Entire sketching activities and sketch 
strokes were recorded to analyse shape transformation using three criteria_-Decomposition, Reinterpretation and Design family_ which were applied to three tasks consisting of short design briefs and initial design stimuli.

\section{Shape rules from the experiment}

As a result of our preliminary experiment, 7 general shape rules (Table 1) and 14 detailed shape rules (Table 2) were identified. These can be regarded as the personal rules of the participants. The hierarchical classification was suggested due to the similarities among shape rules. Note that the outline transformation rule in Table 1 denotes 'changing outline shape including stretching and contour manipulation' while the structure transformation rule indicates 'changing shape position including rotation, translation and symmetry’.

Table 1 General shape rules identified

\begin{tabular}{|c|c|c|c|c|c|c|}
\hline Add & Cut & $\begin{array}{c}\text { Change } \\
\text { view }\end{array}$ & Delete & Outline trans- Substitute & Structure \\
transformation
\end{tabular}

Table 2 Detail shape rules identified

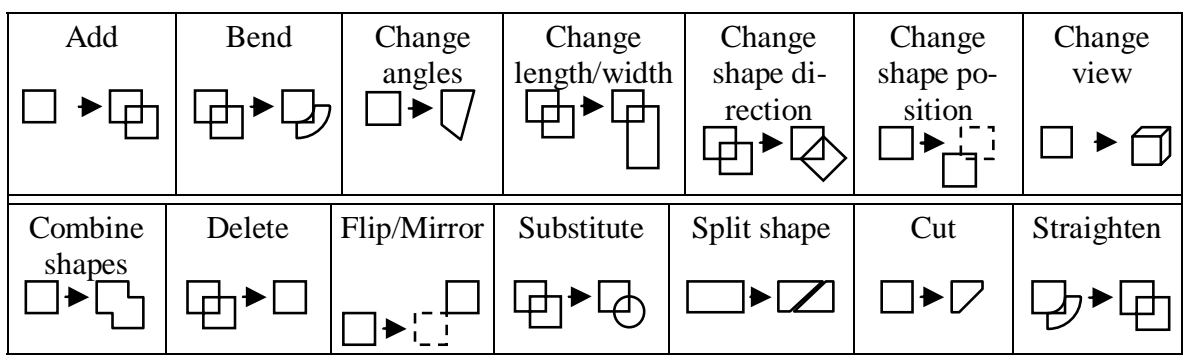

Shape rules in a higher (general) level could contain a number of detailed rules in their lower levels (Figure 1). From our experiment, the outline transformation rule comprises a number of similar shape rules i.e., bend, straighten, change length/width and change angles, while the structure transformation rule includes flip/mirror, change shape direction, split shape, and change shape position rules. The bend rule in the detailed shape rules denotes 'giving curvature to a shape', while the straighten rule indicates the opposite meaning; the change angles rule indicates 'changing an interior angle of a shape'; and the combine shapes rule means 'adding and merging a new shape to an existing shape', while the add rule adds a 
new shape without merging them. Indeed, classification of these shape rules can be further refined; for example, the bend rule can produce different types of curvature to a shape captured in shape rules (e.g. soft radius, sharp radius, a curve with rising curvature and so on).

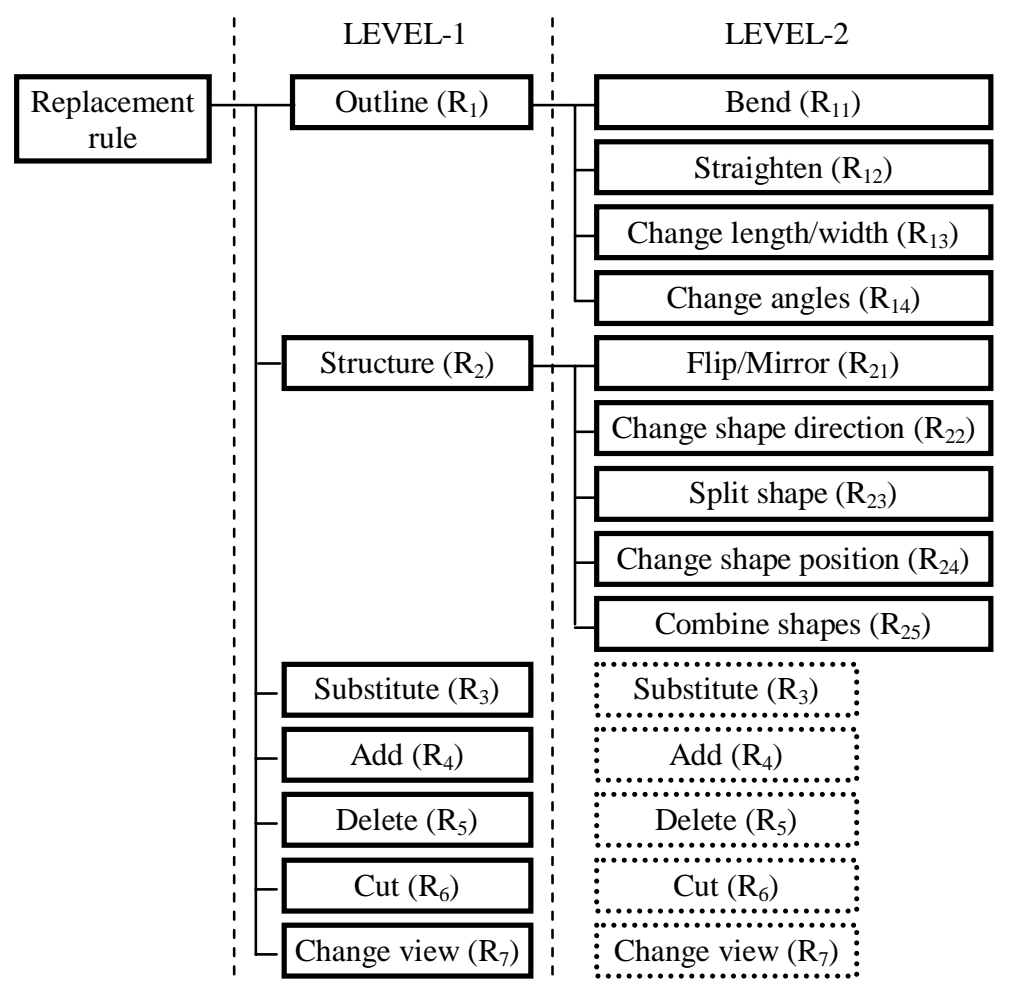

Fig1. Hierarchical classification of the identified shape rules

In addition to providing an objective means of analysis, these rules provide a means for formally generating design alternatives. Note that the graphical representations of these rules express shape transformations in an abstract way and are not meant to represent the exact transformation of a shape, meaning that the same rule may be applied to different shapes and transform them in different ways. For example, the first $\left(R_{12}\right)$ and third $\left(R_{12}\right)$ rules for the design outcome $S 01$ in the Appendix are the same abstract rule — straighten - but are applied under different shape transformations. In addition, this list of rules is not by any means complete; they were, however, sufficient to capture participants' shape transformations. The identified shape rules are then hierarchically classified, and the rules 
$R_{3}$ to $R_{7}$ are directly applied to level-2 because they do not have child rules (Figure 1).

\section{Preference values of shape rules}

Some rules were used by participants significantly more than others, e.g. change shape length/width $\left(R_{12}\right)$, change view $\left(R_{7}\right)$, add $\left(R_{4}\right)$, and straighten $\left(R_{12}\right)$ were used 2 to 10 times more than others (Table 3 ). This result suggests that it may be possible to (i) identify priorities of shape rules, (ii) calculate the preference values for each shape rule based on the frequency of rule use and (iii) use the preference values as speculative tools to provide customisable categorisations of design outcomes. The preference value was calculated by normalisation between 0.0 and 1.0 based on the sum of total use for each rule from the experiment's results (see the last column in Table 3), and the value can be incrementally updated whenever new results from experimentation are added. For example, use of the substitute rule was hardly observed in our experiment; thus the preference value is considered as 0.0. It can be, however, changed depending on the result of additional experiments.

Table 3 The use of the shape rules in architectural design. The numbers in each task indicate the frequency of rule use and the number of participants who used the rule (in parentheses).

\begin{tabular}{|c|l||c|c|c|c|c|}
\hline Rank & \multicolumn{1}{|c||}{ Shape Rules } & Task 1 & Task 2 & Task 3 & $\begin{array}{c}\text { Total } \\
\text { use of } \\
\text { the rule }\end{array}$ & $\begin{array}{c}\text { Preference } \\
\text { value } \\
\text { (normalised) }\end{array}$ \\
\hline \hline 1 & Change length/width $\left(\mathrm{R}_{13}\right)$ & $35(6)$ & $9(4)$ & $11(3)$ & 55 & 0.239130 \\
\hline 2 & Change view $\left(\mathrm{R}_{7}\right)$ & $0(0)$ & $22(6)$ & $21(5)$ & 43 & 0.186957 \\
\hline 3 & Add $\left(\mathrm{R}_{4}\right)$ & $18(4)$ & $8(3)$ & $7(3)$ & 33 & 0.143478 \\
\hline 4 & Straighten $\left(\mathrm{R}_{12}\right)$ & $22(6)$ & $0(0)$ & $2(2)$ & 24 & 0.104348 \\
\hline 5 & Change shape position $\left(\mathrm{R}_{24}\right)$ & $13(5)$ & $0(0)$ & $1(1)$ & 14 & 0.060870 \\
\hline 6 & Bend $\left(\mathrm{R}_{11}\right)$ & $9(2)$ & $0(0)$ & $2(2)$ & 11 & 0.047826 \\
\hline 7 & Delete $\left(\mathrm{R}_{5}\right)$ & $10(2)$ & $1(1)$ & $0(0)$ & 11 & 0.047826 \\
\hline 8 & Change shape direction $\left(\mathrm{R}_{22}\right)$ & $10(5)$ & $0(0)$ & $0(0)$ & 10 & 0.043478 \\
\hline 9 & Combine shapes $\left(\mathrm{R}_{25}\right)$ & $5(3)$ & $4(3)$ & $0(0)$ & 9 & 0.039130 \\
\hline 10 & Split shape $\left(\mathrm{R}_{23}\right)$ & $0(0)$ & $0(0)$ & $8(2)$ & 8 & 0.034783 \\
\hline 11 & Change angles $\left(\mathrm{R}_{14}\right)$ & $5(2)$ & $1(1)$ & $0(0)$ & 6 & 0.026087 \\
\hline 12 & Flip/Mirror $\left(\mathrm{R}_{21}\right)$ & $3(2)$ & $2(1)$ & $0(0)$ & 5 & 0.021739 \\
\hline 13 & Cut $\left(\mathrm{R}_{6}\right)$ & $0(0)$ & $0(0)$ & $1(1)$ & 1 & 0.004348 \\
\hline 14 & Substitute $\left(\mathrm{R}_{3}\right)$ & $0(0)$ & $0(0)$ & $0(0)$ & 0 & 0.0 \\
\hline
\end{tabular}




\section{Formalisation of customised viewpoints}

Design outcomes can be categorised differently depending on customised viewpoints. For example, shapes $S_{1}$ and $S_{2}$, which are generated by a number of shape rules with a sequential manner, e.g. $\left\{S_{1} \mid R_{a}, R_{b}, R_{a}, R_{d}\right\}$ and $\left\{S_{2} \mid R_{a}, R_{c}, R_{e}\right\}$ (Figure 2), can be in the same cluster if the shape rule $R_{a}$ is a most important criterion, while they could be classified in a different cluster in other cases.

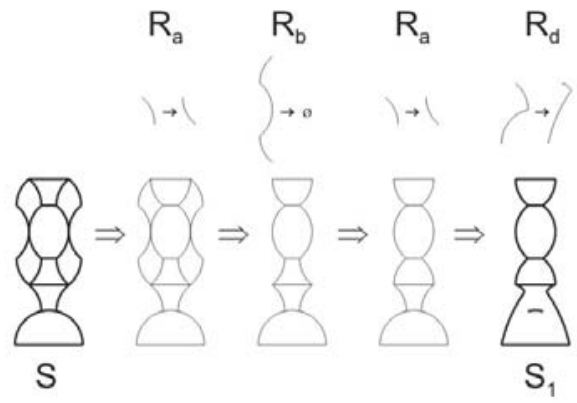

(a)

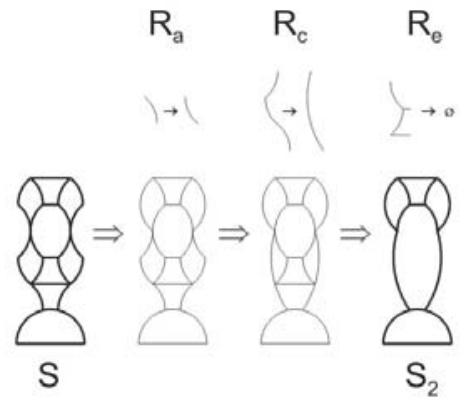

(b)

Fig2. Example shapes $S_{1}$ and $S_{2}$ with respective rule sequences

Here an experimental approach to categorising outcomes is presented which calculates and sorts a preference value for each outcome. The preference value $P$ for each outcome is normalised between 0.0 and 1.0, and can be obtained via two customised viewpoints: (i) absolute $P$ by the frequency of shape rule use; (ii) relative $P$ by shape rule classification level. To calculate the above $P$ for each outcome, a number of criteria need to be predefined: (i) a hierarchical (multi-level) classification of shape rules (Figure 1); (ii) a weight value $Q_{m}$ for each step of a shape rule sequence; (iii) a preference value $V_{R_{n}}$ for each shape rule $R_{n}$ (see the last column in Table 3). For now, each $Q_{m}$ is equally distributed depending on the maximum number of sequence steps from a design outcome, i.e. $Q_{m}=\frac{1}{L}$ where $L$ is the maximum number of sequence steps. The distribution of $Q_{m}$, however, could be adjusted in future research, e.g. the first and last step of a sequence could have more weight if we consider those to be more effective for outcomes than others. The details of the formalisation of $P$ by each viewpoint are described in the following sections. 


\section{Customised viewpoints by absolute preference values}

According to our experimental data [8], designers respond positively to specific rules which can affect the types of design outcomes. In this paper, design outcomes are categorised based on the preference value of shape rules because a preference value offers one way of representing a personal design intention. An absolute $P_{1}$ for the above shape $S_{1}$, which is generated by a four step rule sequence, i.e. $\left\{S_{1} \mid R_{a}, R_{b}, R_{a}, R_{d}\right\}$, is calculated by all the rule values with respective sequential weights. This value can be used to determine the order of outcomes without any classifications, so designers could limit the number of preferable outcomes from the entire set of possible outcomes. Considering the sequence of rule application for the shape $S_{1}$, the absolute $P_{1}$ is calculated as

$$
P_{1}=V_{R_{a}} Q_{1}+V_{R_{b}} Q_{2}+V_{R_{a}} Q_{3}+V_{R_{d}} Q_{4}
$$

Because $Q_{m}$ is equally distributed here, the absolute $P_{1}$ is the same as the sum of $V_{R_{n}}$ divided by the sequence length, and can be summarised as

$$
P_{1}=\left(V_{R_{a}}+V_{R_{b}}+V_{R_{a}}+V_{R_{d}}\right) Q_{m}
$$

Thus, a general shape $S_{1}$ can be calculated as below when $V_{i}$ is the sum of the preference values of used shape rules for shape $S_{1}$ :

$$
P_{i}=V_{i} Q_{m}
$$

\section{Customised viewpoints by relative preference values with a rule classi- fication level}

Sometimes designers may wish to limit outcomes by the generality of shapes $[18,19]$. In this case, setting the outcome criteria by classification levels of shape rules would be useful because a higher classification level of shape rules allows a broad range of shape types while a lower level allows more specific shape types. When a shape rule classification has a depth of $k$, there are $k$ different relative $P$ values, based on the depth in the hierarchy. In this viewpoint, a relative $P_{1}$ for the above shape $S_{1}$ is calculated by the different levels of shape rule classification rather than $V_{R_{n}}$. Consider $V_{R_{n}(k)}$ is the $P$ of the $k$ th level of a shape rule classification that contains $R_{n}$, and is the sum of $V_{R_{n}}$ in the $(k+1)$ th level (Figure 3). Then the relative $P_{1_{k}}$ for the above shape $S_{1}$ by the $k$ th level of shape rule classification is calculated using Equation (2) as 


$$
P_{1_{k}}=\left(V_{R_{a}(k)}+V_{R_{b}(k)}+V_{R_{a}(k)}+V_{R_{d}(k)}\right) Q_{m}
$$

Note that the preference value of each shape rule is the sum of its child rules, e.g. the preference value of $R_{11}$ in the level-2 in Figure 3 is the sum of the preference values of $\left\{R_{111}, R_{112}, R_{113}\right\}$, which are the child rules of $R_{11}$. If $R_{n}$ is located in a lowest level, $V_{R_{n}}$ is applied to $V_{R_{n}(k)}$; thus the relative preference value by lowest level is equal to the absolute preference value.

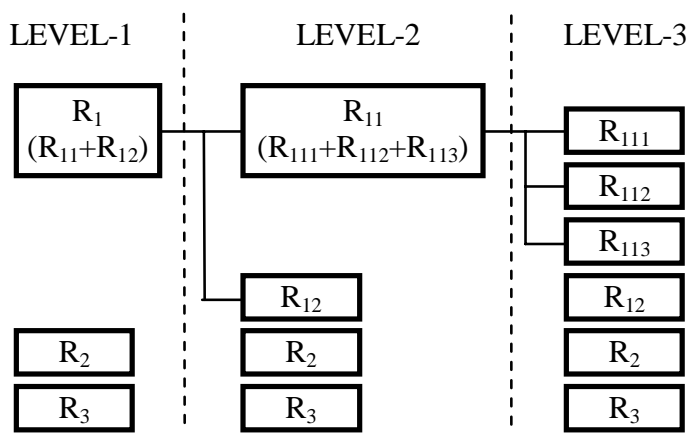

Fig3. Example of the hierarchical classification of preference values

\section{Examples with customised viewpoints}

The selected design outcomes used in this paper were created as a part of our experiment [8]. From the initial shape (a candle holder) we generated 115 outcomes that have a maximum of 10 rule sequences (Table 4 and Figure 4); 95 of the outcomes are derived from the subsequences of the final 20 outcomes (SO1 - S20) while omitting duplicated designs. For example, the outcome $S 05$ in Figure 4 is generated by four sequential rules (see Appendix) which has four possible designs, i.e. $S 05-1$ by $\left\{R_{11}\right\}$, S05-2 by $\left\{R_{11}, R_{11}\right\}$, S05-3 by $\left\{R_{11}, R_{11}, R_{13}\right\}$, and $S 05-4$ by $\left\{R_{11}, R_{11}, R_{13}, R_{5}\right\}$. The rule sequences of $S 05-1$ and $S 05-2$, however, are already generated by $S 03$; thus only two designs SO5-3 and S05-4 are used and a total 115 outcomes are generated. 
Table 4 The initial shape and the twenty final outcomes of the rule sequences

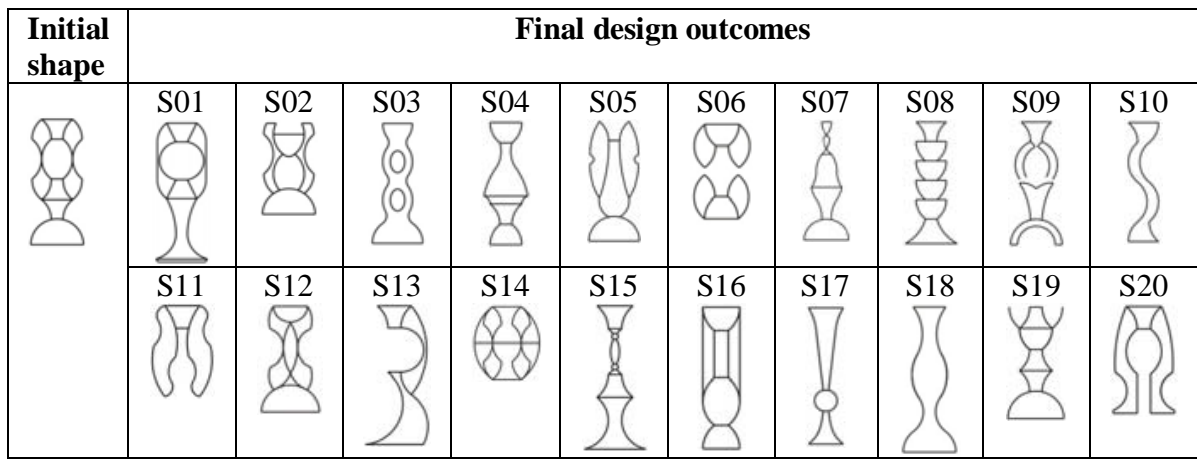

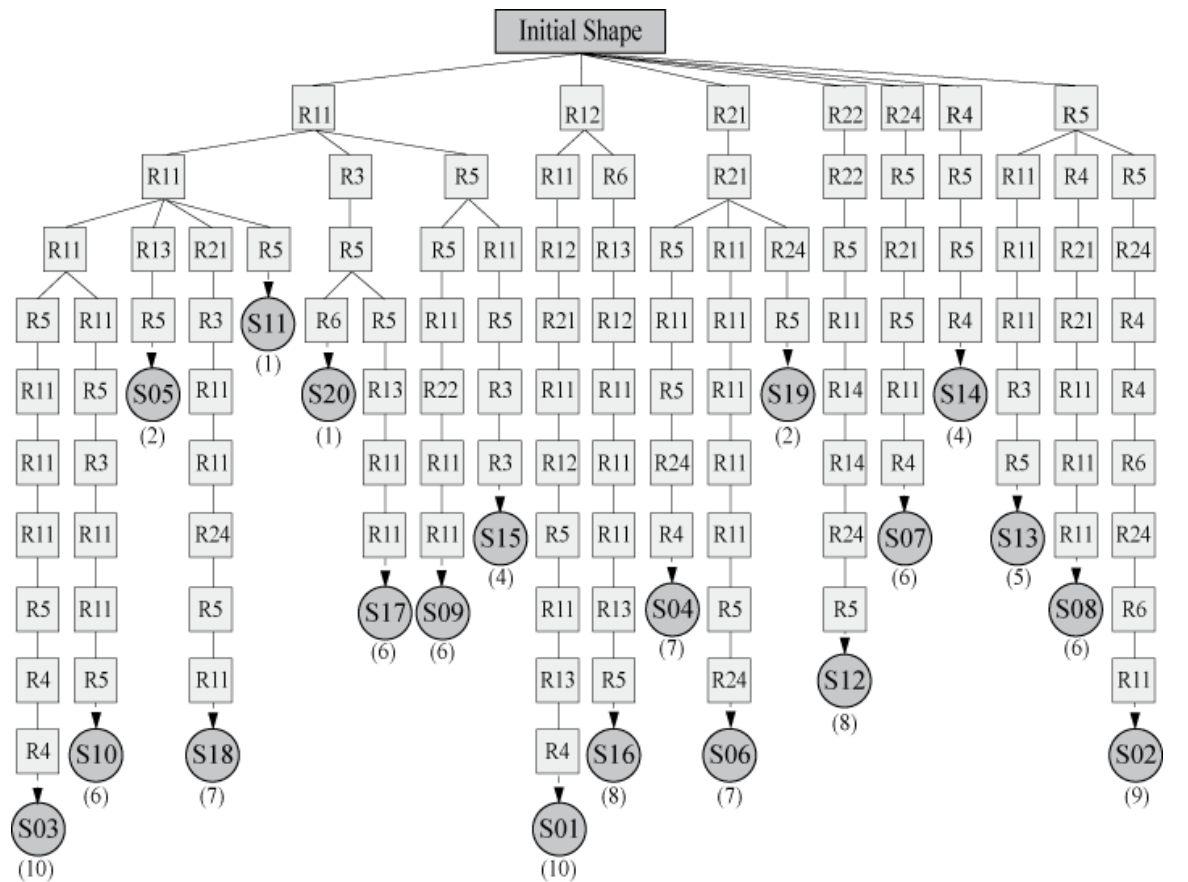

Fig4. Rule sequences of the design outcomes in Table 4. The numbers in parentheses indicate how many outcomes were selected from the subsequences of each final outcome (S01 - S20). See the Appendix for all the design outcomes.

The formalised approach with the two customised viewpoints is respectively evaluated with the above examples. In this evaluation, we analysed 20 outcomes (top 10 and bottom 10) by each viewpoint from the total of 115 outcomes. All the preference values of the outcomes are calculated by 
two customised viewpoints: (i) absolute $P$, which is equal to the relative $P$ with rule classification level-2 in this paper (Tables 5 and 6), and (ii) relative $P$ with rule classification level-1 (Tables 7 and 8). Note that since the categorisations are focused on the manner of generating designs the results may not be dependent on shape similarity; however, they are considered as the designs most likely to be produced by designers with particular preferences.

\section{Outcomes by absolute preference values}

Based on the absolute $P$ for each rule, the outcomes were sorted as shown in Tables 5 and 6. Because the preference values are likely the design intentions of the experiment's participants, they cannot reflect general deisgn preferences. This, however, can be an alternative way to support personal preferences, as previously described.

Table 5 Top 10 and bottom 10 outcomes based on absolute preference values

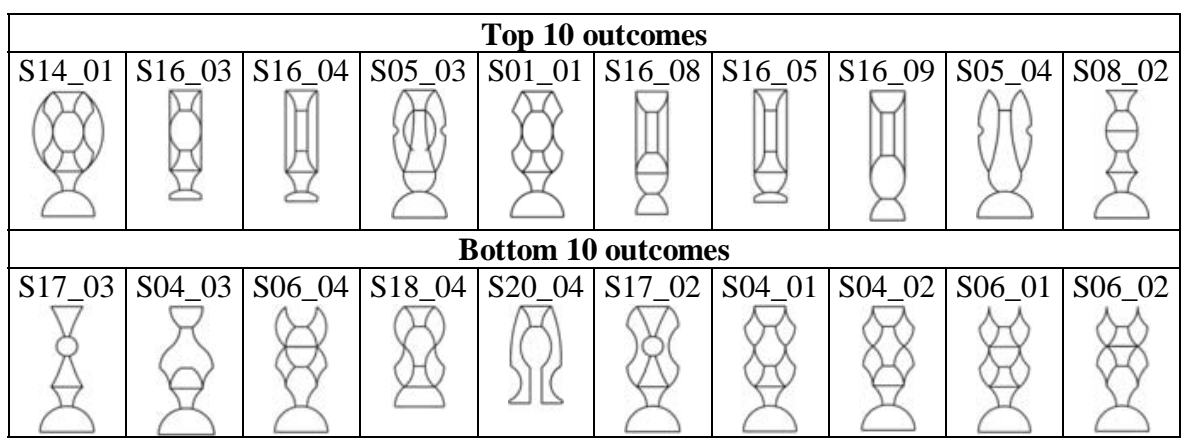

Table 6 Respective absolute preference values $(\mathrm{P})$ and rule sequences of the outcomes in Table 5

\begin{tabular}{|c|l|l|l|}
\hline Rank & Outcomes & \multicolumn{1}{|c|}{ P } & \multicolumn{1}{c|}{ Applied rule sequence } \\
\hline \hline 1 & $\mathrm{~S} 14 \_01$ & 0.1434779 & $\mathrm{R}_{4}$ \\
\hline 2 & $\mathrm{~S} 16 \_03$ & 0.115942 & $\mathrm{R}_{12}-\mathrm{R}_{6}-\mathrm{R}_{13}$ \\
\hline 3 & $\mathrm{~S} 16 \_04$ & 0.1130435 & $\mathrm{R}_{12}-\mathrm{R}_{6}-\mathrm{R}_{13}-\mathrm{R}_{12}$ \\
\hline 4 & $\mathrm{~S} 05 \_03$ & 0.111594 & $\mathrm{R}_{11}-\mathrm{R}_{11}-\mathrm{R}_{13}$ \\
\hline 5 & $\mathrm{~S} 01 \_01$ & 0.104348 & $\mathrm{R}_{12}$ \\
\hline 6 & $\mathrm{~S} 16 \_08$ & 0.1043478 & $\mathrm{R}_{12}-\mathrm{R}_{6}-\mathrm{R}_{13}-\mathrm{R}_{12}-\mathrm{R}_{11}-\mathrm{R}_{11}-\mathrm{R}_{11}-\mathrm{R}_{13}$ \\
\hline 7 & $\mathrm{~S} 16 \_05$ & 0.1 & $\mathrm{R}_{12}-\mathrm{R}_{6}-\mathrm{R}_{13}-\mathrm{R}_{12}-\mathrm{R}_{11}$ \\
\hline 8 & $\mathrm{~S} 1609$ & 0.0980676 & $\mathrm{R}_{12}-\mathrm{R}_{6}-\mathrm{R}_{13}-\mathrm{R}_{12}-\mathrm{R}_{11}-\mathrm{R}_{11}-\mathrm{R}_{11}-\mathrm{R}_{13}-\mathrm{R}_{5}$ \\
\hline \multirow{2}{*}{9} & $\mathrm{~S} 05 \_04$ & 0.095652 & $\mathrm{R}_{11}-\mathrm{R}_{11}-\mathrm{R}_{13}-\mathrm{R}_{5}$ \\
\cline { 2 - 4 } & $\mathrm{S} 08 \_02$ & 0.095652 & $\mathrm{R}_{5}-\mathrm{R}_{4}$ \\
\hline \multirow{2}{*}{106} & $\mathrm{~S} 17 \_03$ & 0.031884 & $\mathrm{R}_{11}-\mathrm{R}_{3}-\mathrm{R}_{5}$ \\
\hline \multirow{2}{*}{107} & $\mathrm{~S} 04 \_03$ & 0.0304347 & $\mathrm{R}_{21}-\mathrm{R}_{21}-\mathrm{R}_{5}$ \\
\cline { 2 - 5 } & $\mathrm{S} 06 \_04$ & 0.0304347 & $\mathrm{R}_{21}-\mathrm{R}_{21}-\mathrm{R}_{11}-\mathrm{R}_{11}$ \\
\hline
\end{tabular}




\begin{tabular}{|l|l|l|l|}
\hline 109 & $\mathrm{~S} 18 \_04$ & 0.0293478 & $\mathrm{R}_{11}-\mathrm{R}_{11}-\mathrm{R}_{21}-\mathrm{R}_{3}$ \\
\hline 110 & $\mathrm{~S} 20 \_04$ & 0.025 & $\mathrm{R}_{11}-\mathrm{R}_{3}-\mathrm{R}_{5}-\mathrm{R}_{6}$ \\
\hline 111 & $\mathrm{~S} 17 \_02$ & 0.023913 & $\mathrm{R}_{11}-\mathrm{R}_{3}$ \\
\hline \multirow{4}{*}{112} & $\mathrm{~S} 04 \_01$ & 0.021739 & $\mathrm{R}_{21}$ \\
\cline { 2 - 4 } & S04_02 & 0.021739 & $\mathrm{R}_{21}-\mathrm{R}_{21}$ \\
\cline { 2 - 4 } & $\mathrm{S} 06 \_01$ & 0.021739 & $\mathrm{R}_{21}$ \\
\cline { 2 - 4 } & S06_02 & 0.021739 & $\mathrm{R}_{21}-\mathrm{R}_{21}$ \\
\hline
\end{tabular}

\section{Outcomes by relative preference values}

Unlike the outcomes by absolute $P$, the outcomes by relative $P$ with the rule classification level-1 show a visible classification in their rule sequences (Tables 7 and 8).

Table 7 Top 10 and bottom 10 outcomes using relative preference values with rule classification level-1

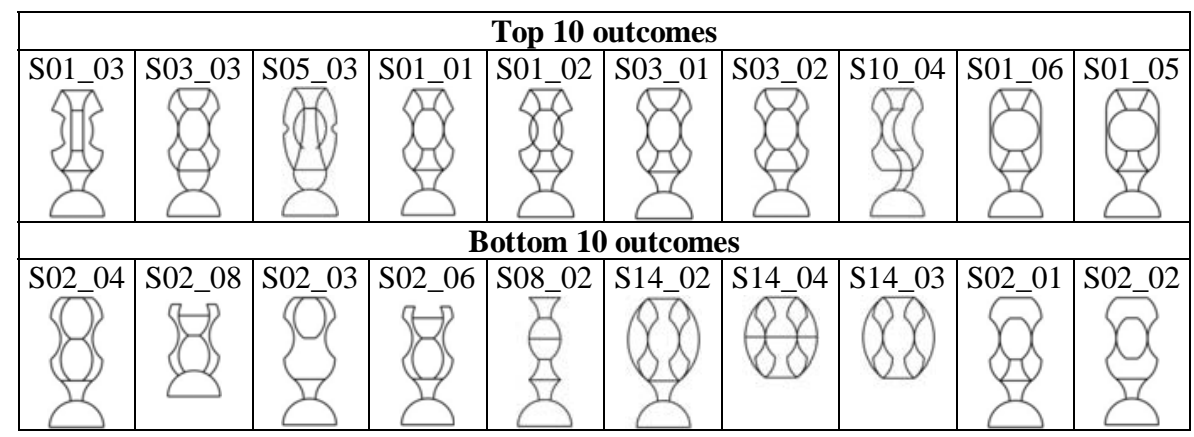

For the top 10 outcomes, the first eight (where the preference value is $0.417391)$ are generated using the outline rule family $R_{1}\left(R_{11}-R_{14}\right)$ only, while the remaining two are generated using mixed rules, i.e. they have one structure rule $\left(R_{21}\right)$ from the $R_{2}$ family as well. For the bottom 10 outcomes, the most frequently used shape rules are delete $\left(R_{5}\right)$, add $\left(R_{4}\right)$ and change shape position $\left(R_{24}\right)$. Although the absolute $P$ for add $\left(R_{4}\right)$ is the third biggest value (Table 3 ), the $R_{4}$ rule is considered as the rules that have the lowest $P$ in this viewpoint. This is because (i) some rules that have the lowest $P$ such as change angles $\left(R_{14}\right)$ and flip/mirror $\left(R_{21}\right)$ are classified as outline $\left(R_{1}\right)$ and structure $\left(R_{2}\right)$ rules respectively and they are the two largest $P$, and (ii) cut $\left(R_{6}\right)$ and substitute $\left(R_{3}\right)$ rules were rarely used in the 115 outcomes. The result seems well suited to the purpose, i.e. identifying outcomes that share the same rule classifications. 
Categorisation of designs according to preference values for shape rules 13

Table 8 Respective relative preference values $(\mathrm{P})$ and rule sequences of the outcomes in Table 7

\begin{tabular}{|c|c|c|c|}
\hline Rank & Outcomes & $\mathbf{P}$ & Applied rule sequence \\
\hline \multirow{3}{*}{1} & "S01_03 & 0.417391 & $\mathrm{R}_{12}-\mathrm{R}_{11}-\mathrm{R}_{12}$ \\
\hline & S03_03 & 0.417391 & $\mathrm{R}_{11}-\mathrm{R}_{11}-\mathrm{R}_{11}$ \\
\hline & S05_03 & 0.417391 & $\mathrm{R}_{11}-\mathrm{R}_{11}-\mathrm{R}_{13}$ \\
\hline \multirow{5}{*}{4} & S01_01 & 0.4173909 & $\mathrm{R}_{12}$ \\
\hline & S01_02 & 0.4173909 & $\mathrm{R}_{12}-\mathrm{R}_{11}$ \\
\hline & S03_01 & 0.4173909 & $\mathrm{R}_{11}$ \\
\hline & S03_02 & 0.4173909 & $\mathrm{R}_{11}-\mathrm{R}_{11}$ \\
\hline & S10_04 & 0.4173909 & $\mathrm{R}_{11}-\mathrm{R}_{11}-\mathrm{R}_{11}-\mathrm{R}_{11}$ \\
\hline 9 & S01_06 & 0.381159 & $\mathrm{R}_{12}-\mathrm{R}_{11}-\mathrm{R}_{12}-\mathrm{R}_{21}-\mathrm{R}_{11}-\mathrm{R}_{12}$ \\
\hline 10 & S01_05 & 0.373913 & $\mathrm{R}_{12}-\mathrm{R}_{11}-\mathrm{R}_{12}-\mathrm{R}_{21}-\mathrm{R}_{11}$ \\
\hline 106 & S02_04 & 0.1097825 & $\mathrm{R}_{5}-\mathrm{R}_{5}-\mathrm{R}_{24}-\mathrm{R}_{4}$ \\
\hline 107 & S02_08 & 0.098913 & $R_{5}-R_{5}-R_{24}-R_{4}-R_{4}-R_{6}-R_{24}-R_{6}$ \\
\hline 108 & S02_03 & 0.0985507 & $\mathrm{R}_{5}-\mathrm{R}_{5}-\mathrm{R}_{24}$ \\
\hline 109 & S02_06 & 0.097826 & $R_{5}-R_{5}-R_{24}-R_{4}-R_{4}-R_{6}$ \\
\hline \multirow{3}{*}{110} & S08_02 & 0.095652 & $\mathrm{R}_{5}-\mathrm{R}_{4}$ \\
\hline & S14_02 & 0.095652 & $\mathrm{R}_{4}-\mathrm{R}_{5}$ \\
\hline & S14_04 & 0.095652 & $R_{4}-R_{5}-R_{5}-R_{4}$ \\
\hline 113 & S14_03 & 0.07971 & $\mathrm{R}_{4}-\mathrm{R}_{5}-\mathrm{R}_{5}$ \\
\hline \multirow{2}{*}{114} & S02_01 & 0.047826 & $\mathrm{R}_{5}$ \\
\hline & S02_02 & 0.047826 & $\mathrm{R}_{5}-\mathrm{R}_{5}$ \\
\hline
\end{tabular}

\section{Discussion}

The design examples used in this paper attempt to reflect the kind of shapes and shape transformations used in the conceptual stage of design, where designs tend to be vague and ambiguous. For this reason, shape rules that express transformations of a shape in an abstract way without representing an exact transformation of the shape have been used as previously mentioned. As an extension of the presented approach for categorisation of designs, use in later stages of design would require more detailed shape rules. For example, change length/width $\left(R_{13}\right)$ could be detailed with definitions of length and width, and with proportional rate of change. Additionally, a preference value for a single rule could be extended to certain lengths of rule sequences, e.g. a preference value for the rule sequence $\left\{R_{1}, R_{2}, R_{3}\right\}$ could support more in-depth personal preferences in a shape generation process.

On the other hand, we also tested another viewpoint regarding the complexity of outcomes based on multiple criteria: (i) the length of a rule sequence; (ii) the number of shape rules used; and (iii) the complexity type of a shape rule. The complexity type was determined by whether it con- 
tributes to the complexity of outcomes. For example, the add $\left(R_{4}\right)$ rule increases complexity, the delete $\left(R_{5}\right)$ rule decreases it, but other rules do not affect complexity. The result of the complexity viewpoint, however, was not very usable. It seems the length of a rule sequence does not affect the complexity of design outcomes. Instead, there might be more crucial criteria to determine the complexity of outcomes such as the combination of used rules, and different weightings for each step of shape rule sequence, etc.

Currently, the suggested approach is designed as a post-categorisation method after generating designs. As it seems that generating sequences of designs that are aligned with design intentions could effectively reduce design spaces [21], we may need to adapt our approach as a precategorisation method, which defines personal design intentions before generating designs.

\section{Conclusion}

The experimental approach that uses a hierarchical classification of shape rules with preference value of each shape rule offers multiple ways of categorising outcomes depending on designers' needs. A preference value of each design outcome, which is used as a speculative tool to identify personal preference of shape generation, has been defined via two criteria, i.e. (i) an absolute preference value based on the frequency of rule use, and (ii) a relative preference value based on shape rule classification levels. A hierarchical classification of shape rules and a preference value for each shape rule in this paper have been identified from the preliminary experiment, and the examples from our experiment are used to evaluate the proposed approach.

The result of categorised outcomes with the worked examples reveals the possibility of providing more preferable and refined outcomes to designers. Therefore, this work illuminates a phenomenon that might be the subject of future research of the current project, and reveals potential diversity in the exploitation of shape grammar systems. Future work is concerned with detailing abstracted rule transformation using exact shape expression, adding a criterion regarding complexity of outcomes, applying the approach as a pre-categorisation method, and exploring how these results can inform the development of computational tools intended to support conceptual design. 
Categorisation of designs according to preference values for shape rules 15

\section{Acknowledgements}

This work has been carried out as a part of the project "Design Synthesis and Shape Generation" funded under the AHRC 'Designing for the 21st Century' programme. The authors would like to thank the designers and researchers who participated in the study for their cooperation.

\section{References}

1. Bentley PJ (1999) Aspects of evolutionary design by computers, in Advances in Soft Computing - Engineering design and manufacturing, Roy R, Furuhashi T, Editors. Springer-Verlag, London, UK, 99-118

2. Stiny G (2006) Shape: Talking about Seeing and Doing, Cambridge, Mass, MIT Press

3. Antonsson EK, Cagan J (2001) Formal engineering design synthesis. 2001, Cambridge, UK, Cambridge University Press

4. Stiny G (1980) Introduction to shape and shape grammars, Environment and Planning B, 7:343-351

5. Chase SC (2002) A model for user interaction in grammar-based design systems, Automation in Construction, 11:161-172

6. Piazzalunga U, Fitzhorn P (1998) Note on a three-dimensional shape grammar interpreter, Environment and Planning B: Planning and Design, 25:11-30

7. Knight TW (1996) Shape grammars: five questions. Environment and Planning B: planning and Design, 26:4:477 - 501

8. Lim S, Prats M, Chase S, et al. (2008) Sketching in Design: Formalising a transformational process, in Computer Aided Architectural Design and Research in Asia (CAADRIA'08), Chiang Mai, Thailand

9. Mckay A, Jowers I, Chau HH, et al. (2008) Computer aided design: an early shape synthesis system, in International Conference in Advanced Engineering Design And Manufacture (ICADAM), Sanya, China

10. Mahr ML, Balachandran B, Zhang DM (1995) Case-based Reasoning in Design, New Jersey, Lawrence Erlbaum Associates

11. Bailey A, Harris C (1999) Using Hierarchical Classification to Exploit Context in Pattern Classification for Information Fusion, in Proceedings of the Second International Conference on Information Fusion

12. Michalski RS, Stepp RE (1983) Automated construction of classifications: Conceptual clustering versus numerical taxonomy, in IEEE Transactions on Pattern Analysis and Machine Intelligence, PAMI-5

13. Rammal R, Toulouse G, Virasoro MA (1986) Ultrametricity for physicists, Reviews of Modern Physics, 58:765-788

14. Reich Y, Fenves SJ (1991) The Formation and use of Abstract Concepts in Design, in Concept Formation: Knowledge and Experience in Unsupervised Learning, Fisher DH, Pazzani MJ, Editors, Morgan Kaufmann, Los Altos, CA, 323-353 
15. Fisher D, Xu L, Zard N (1992) Ordering effects in clustering, in Proceedings of the Ninth International Conference on Machine Learning, San Mateo, CA, Morgan Kaufmann

16. Gordon AD (1996) Hierarchical Classification, in Clustering and Classification, Arabie P, Hubert LJ, Soete GD, Editors, World Scientific Publishing, 65121

17. Howard-Jones PA (1998) The variation of ideational productivity over short timescales and the influence of an instructional strategy to defocus attention, in Proceedings of Twentieth Annual Meeting of the Cognitive Science Society, Hillsdale, New Jersey, Lawrence Erlbaum Associates

18. Duffy AHB, Kerr SM (1993) Customised Perspectives of past designs from automated group rationalisations, International Journal of Artificial Intelligence in Engineering, Special Issue on Machine Learning in Design, 8:3:183200

19. Manfaat D, Duffy AHB, Lee BS, (1998) SPIDA: Abstracting and generalising layout design cases, Artificial Intelligence for Engineering Design, Analysis and Manufacturing (AI EDAM), 12:141-159

20. Lim S, Lee BS, Duffy AHB, Incremental modelling of ambiguous geometric ideas (I-MAGI), International Journal of Artificial Intelligence in Engineering, 15:2:93-108

21. Prats M, Earl C (2006) Exploration through drawings in the conceptual stage of product design, in Design Computing and Cognition DCC'06, Gero JS, Editor, Springer, Eindhoven, Netherlands, 83-102

\section{Appendix}

The sequential rule processes of the design outcomes selected in the evaluation section are depicted in this appendix to help the reader's understanding.

\section{Design Outcome - S01}

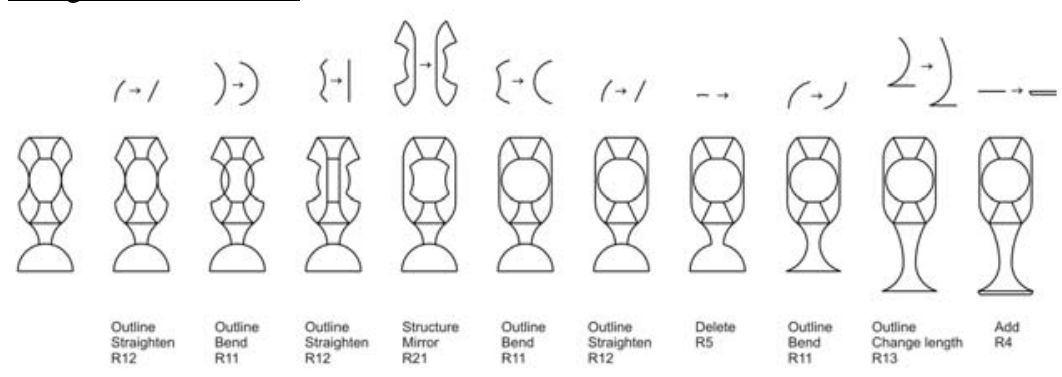


Categorisation of designs according to preference values for shape rules 17 Design Outcome - S02

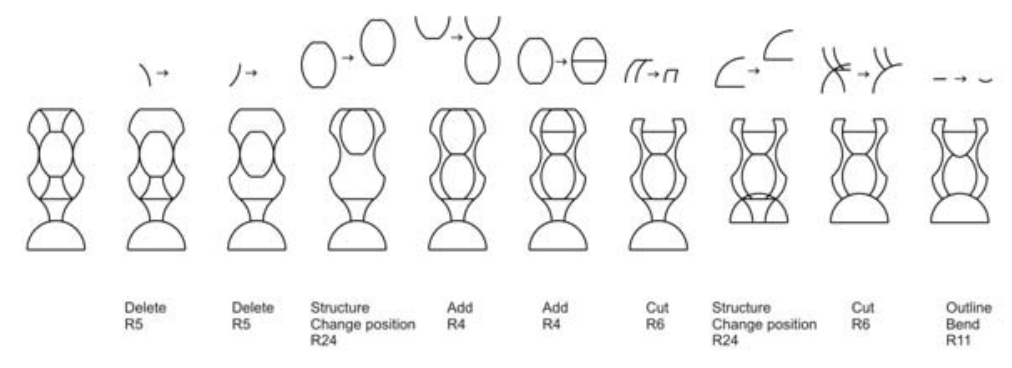

Design Outcome - S03

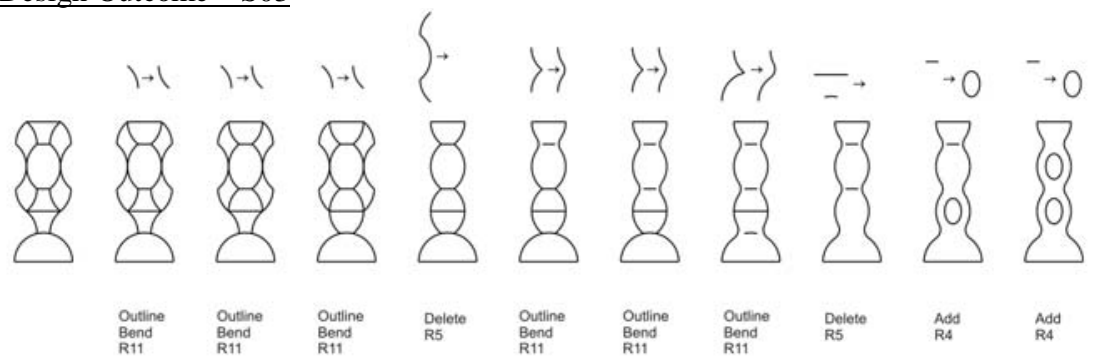

Design Outcome - S04

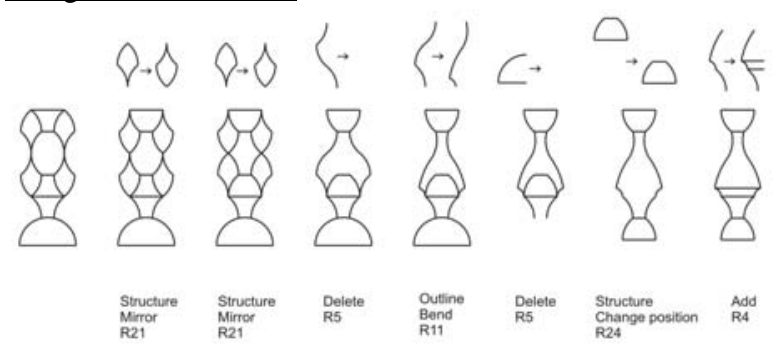

Design Outcome - S05

Design Outcome - S07<smiles>CCC1CCC2C=CC(CCC2)C1C</smiles>

$\left.\theta_{0} \rightarrow \gamma^{8} \rightarrow \&\right\}<$
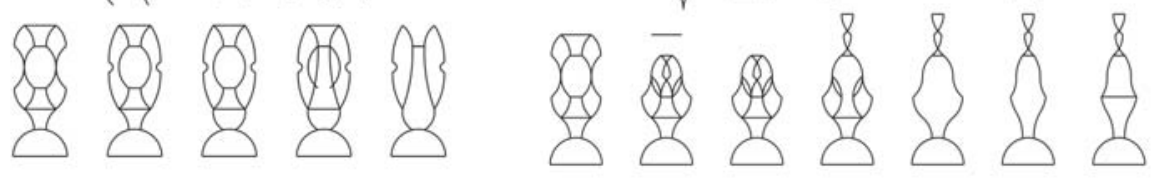

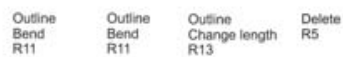

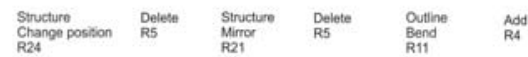


Design Outcome - S06

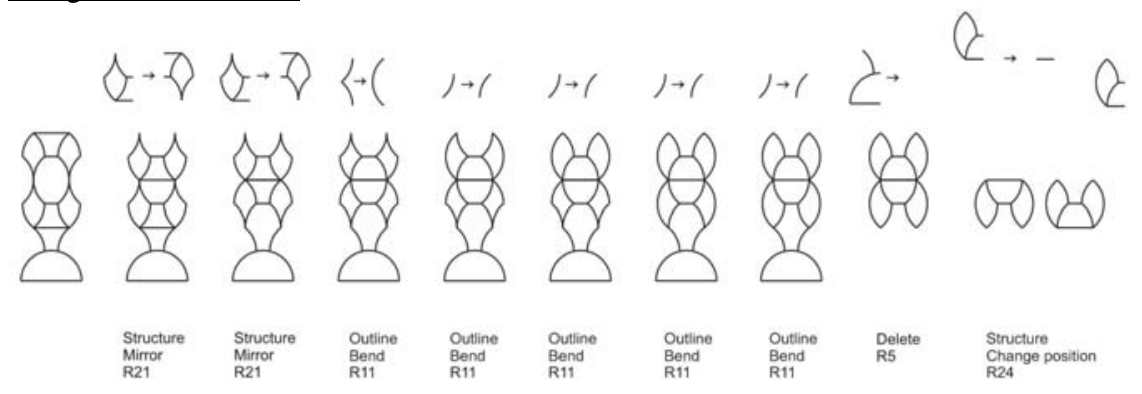

Design Outcome - S08

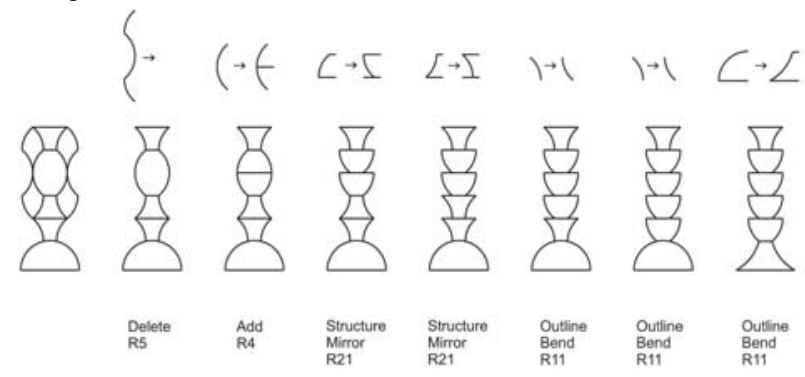

Design Outcome - S09

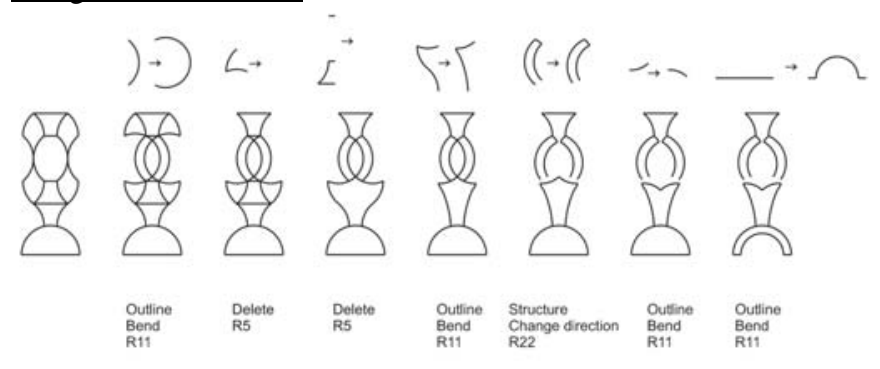

Design Outcome - S10

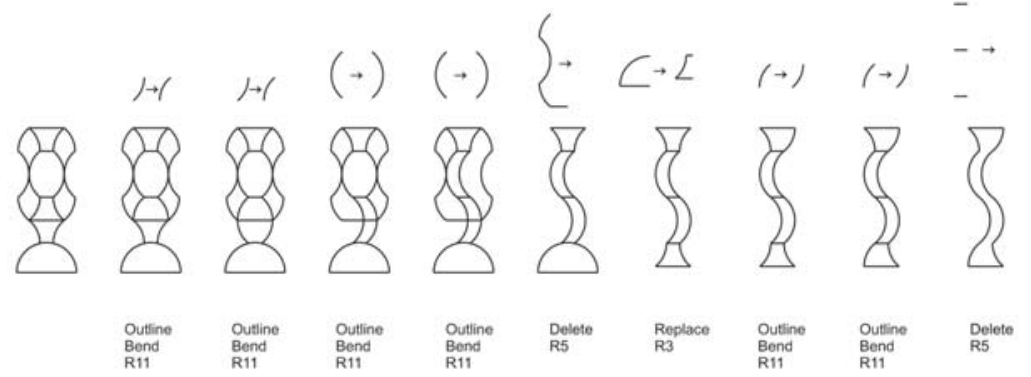


Categorisation of designs according to preference values for shape rules 19

Design Outcome - S11

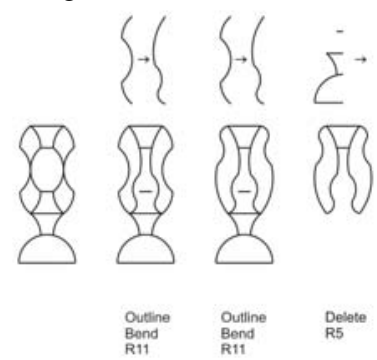

Design Outcome - S14

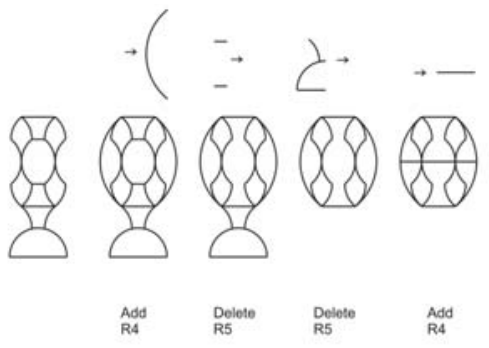

Design Outcome - S12

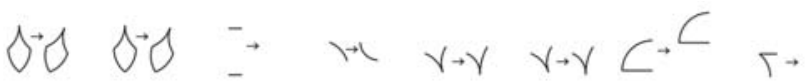

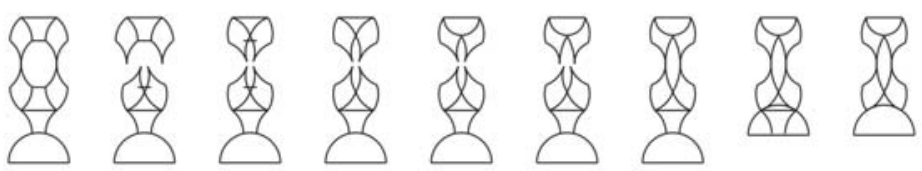

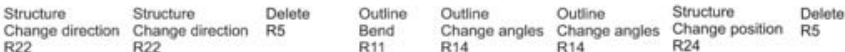

Design Outcome - S13

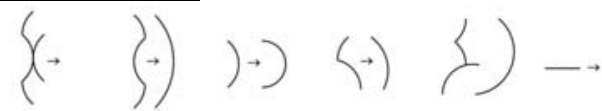

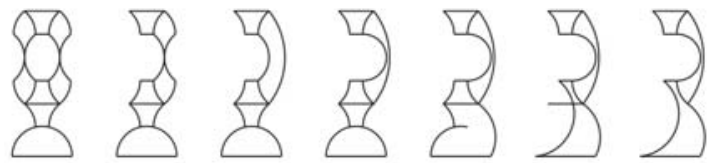

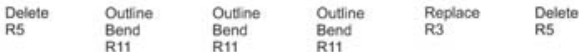

Design Outcome - S15

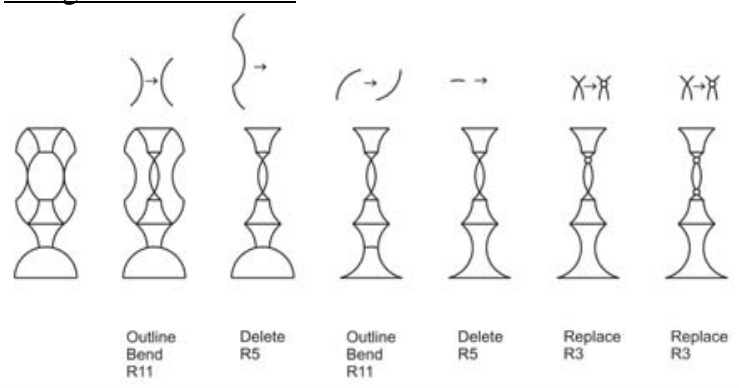


$\underline{\text { Design Outcome - S16 }}$

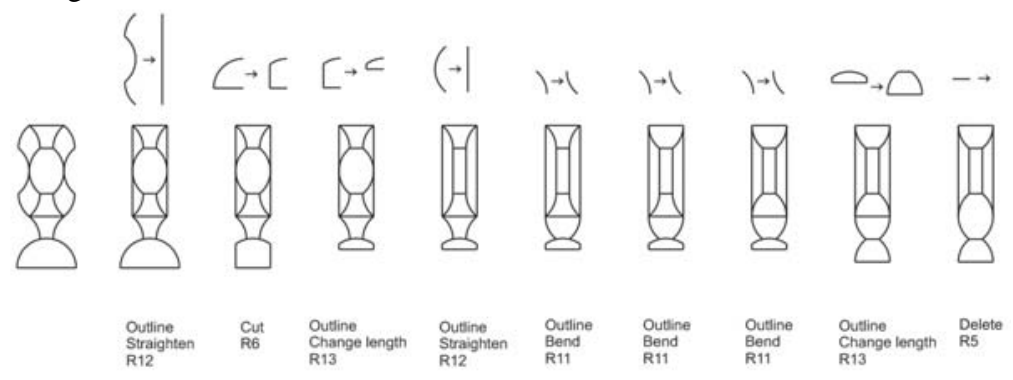

Design Outcome - S17

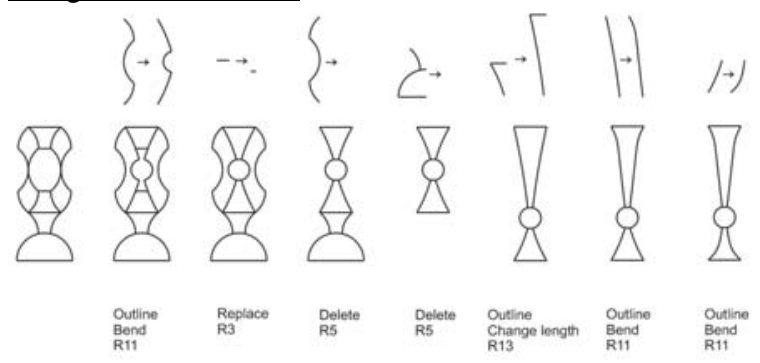

Design Outcome - S18

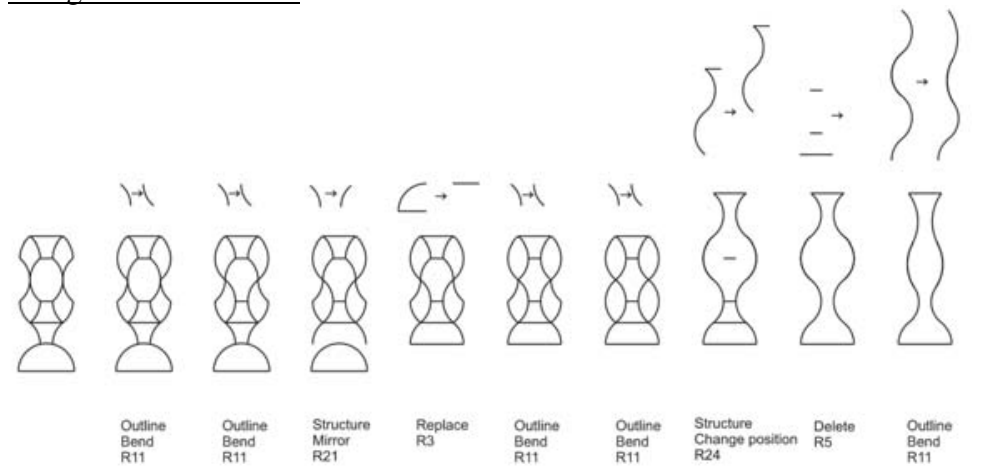

Design Outcome - S19

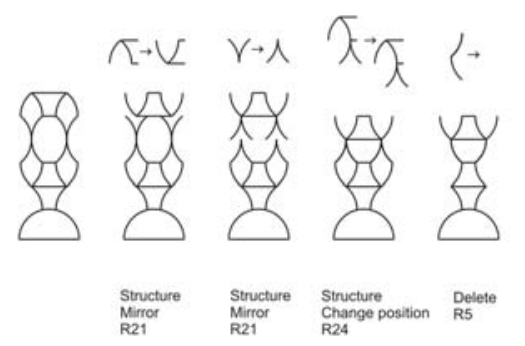

Design Outcome - S20

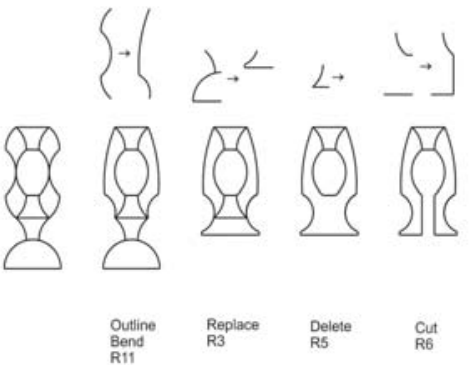

